\title{
Occurrence and location of concentrated load and generator regions observed by Cluster in the plasma sheet
}

\author{
M. Hamrin ${ }^{1}$, P. Norqvist ${ }^{1}$, O. Marghitu ${ }^{2}$, S. Buchert ${ }^{3}$, B. Klecker ${ }^{4}$, L. M. Kistler ${ }^{5}$, and I. Dandouras ${ }^{6}$ \\ ${ }^{1}$ Department of Physics, Umeå University, Umeå, Sweden \\ ${ }^{2}$ Institute for Space Sciences, Bucharest, Romania \\ ${ }^{3}$ Swedish Institute of Space Physics, Uppsala, Sweden \\ ${ }^{4}$ Max-Planck-Institut für extraterrestrische Physik, Garching, Germany \\ ${ }^{5}$ Space Science Center, University of New Hampshire, Durham, USA \\ ${ }^{6}$ CESR-CNRS, Toulouse, France
}

Received: 11 June 2009 - Revised: 21 September 2009 - Accepted: 21 October 2009 - Published: 4 November 2009

\begin{abstract}
Here, and in a companion paper by Hamrin et al. (2009) [Scale size and life time of energy conversion regions observed by Cluster in the plasma sheet], we investigate localized energy conversion regions (ECRs) in the Earth's plasma sheet. In total we have studied 151 ECRs within $660 \mathrm{~h}$ of plasma sheet data from the summer and fall of 2001 when Cluster was close to apogee at an altitude of about $15-20 R_{E}$. Cluster offers appropriate conditions for the investigation of energy conversion by the evaluation of the power density, $\boldsymbol{E} \cdot \boldsymbol{J}$, where $\boldsymbol{E}$ is the electric field and $\boldsymbol{J}$ the current density. From the sign of the power density, we have identified more than three times as many Concentrated Load Regions (CLRs) as Concentrated Generator Regions (CGRs). We also note that the CLRs appear to be stronger. To our knowledge, these are the first in situ observations confirming the general notion of the plasma sheet, on the average, behaving as a load. At the same time the plasma sheet appears to be highly structured, with energy conversion occurring in both directions between the fields and the particles. From our data we also find that the CLRs appear to be located closer to the neutral sheet, while CGRs prefer locations towards the plasma sheet boundary layer (PSBL). For both CLRs and CGRs, $\boldsymbol{E}$ and $\boldsymbol{J}$ in the GSM $y$ (cross-tail) direction dominate the total power density, even though the $z$ contribution occasionally can be significant. The prevalence of the y-direction seems to be weaker for the CGRs, possibly related to a higher fluctuation level near the PSBL.
\end{abstract}

Keywords. Magnetospheric physics (Auroral phenomena; Magnetosphere-ionosphere interactions; Plasma sheet)

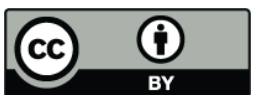

Correspondence to: M. Hamrin (hamrin@space.umu.se)

\section{Introduction}

Energy conversion within the plasma sheet plays an important role for the Earth's magnetosphere and its energy budget. Local energy conversion can be investigated from in situ data by the evaluation of the power density $\boldsymbol{E} \cdot \boldsymbol{J}$, where $\boldsymbol{E}$ is the electric field and $\boldsymbol{J}$ the current density.

A theoretical motivation for the investigation of the quantity $\boldsymbol{E} \cdot \boldsymbol{J}$ can be found e.g. within standard magnetohydrodynamic theory. Assuming a scalar pressure $p$ and using the equation of motion from one-fluid theory, we obtain an equation for the energy conservation of the bulk motion by multiplying with the plasma bulk velocity, $\boldsymbol{v}$,

$\frac{\partial W_{k}}{\partial t}=-\nabla \cdot\left(W_{k} \boldsymbol{v}\right)-\nabla p \cdot \boldsymbol{v}+\boldsymbol{E} \cdot \boldsymbol{J}$,

where $W_{k}$ is the bulk kinetic energy density. The right hand side of the equation corresponds to the source terms for the bulk kinetic energy density: the divergence of the bulk kinetic energy flux, $-\nabla \cdot\left(W_{k} \boldsymbol{v}\right)$, the work done by the pressure forces on the plasma, $-\nabla p \cdot v$, and the work done by the electromagnetical forces on the plasma, $\boldsymbol{E} \cdot \boldsymbol{J}$. When $\boldsymbol{E} \cdot \boldsymbol{J}$ (or $-\nabla p \cdot v)$ is positive, work is done on the plasma and the kinetic energy increases. On the other hand, if $\boldsymbol{E} \cdot \boldsymbol{J}$ is negative, the particles are losing energy to the electromagnetic field, as also can be seen from the Poynting theorem (equation of energy conservation for the electromagnetic field)

$\frac{\partial W_{E M}}{\partial t}=-\nabla \cdot \boldsymbol{S}-\boldsymbol{E} \cdot \boldsymbol{J}$

where $W_{E M}$ is the electromagnetic energy density and $\boldsymbol{S}$ is the Poynting vector. The term $\boldsymbol{E} \cdot \boldsymbol{J}$ hence describes the energy transfer between the particles and the fields.

Local conversion from mechanical (plasma bulk and thermal energy) to electromagnetic energy occurs in generator

Published by Copernicus Publications on behalf of the European Geosciences Union. 
regions where $\boldsymbol{E} \cdot \boldsymbol{J}<0$. The process is reversed in load regions where $\boldsymbol{E} \cdot \boldsymbol{J}>0$ and electromagnetic energy is converted back into mechanical energy by reversible and/or irreversible processes. Note that the term "generator" in the literature can be associated with the generation of electromagnetic energy as well as field-aligned currents. In this investigations we focus on the generation of electromagnetic energy.

On the average, the plasma sheet behaves as a load due to the large scale dawn-dusk electric field and cross-tail current, resulting in a power density $\boldsymbol{E} \cdot \boldsymbol{J}>0$ on the average. On the other hand, at the interface between a near-dipolar magnetic field and the near-Earth plasma sheet with more stretched field lines, plasma convects on average against a pressure gradient providing a generator. This may play an important role for the onset of substorms and auroral arcs at the poleward edge of the auroral oval (Haerendel, 2009). On a smaller scale, the plasma sheet is highly inhomogeneous and energy is converted back and forth between the fields and particles. For example, the plasma sheet magnetically maps to the nightside auroral region. Generator regions in the plasma sheet are hence expected to play an important role for the generation of auroras. The auroral generators have been suggested to be located in various regions in the middle or outer magnetosphere, for example, the low-latitude boundary layer, the plasma sheet and the plasma sheet boundary layer. Several studies have addressed the auroral generator by using analytical (e.g. Rostoker and Boström, 1976), semianalytical (e.g. Lysak, 1985; Vogt et al., 1999), and numerical tools (e.g. Birn and Hesse, 1996; Birn et al., 1996). Lu et al. (2000) made attempts to determine the auroral generator location by magnetic field line mapping between the auroral ionosphere and the outer magnetosphere. Various generator mechanisms and generator locations have also been discussed in Borovsky (1993).

The energy release, transport and conversion in the magnetotail have recently been investigated in a large scale resistive MHD simulation by Birn and Hesse (2005). The simulation shows evidence of the complicated nature of the plasma sheet and tail region, hosting both load and generator regions. The picture is complicated even more by time variations. The simulations of Birn and Hesse (2005) show that energy in the plasma sheet boundary regions is converted back and forth between particles and fields. The oscillation period between loads and generators in this region is of the order 4 min which results in a life time of $\sim 2 \mathrm{~min}$ for loads and generators, respectively.

However, there is in general a lack of in situ investigations of the energy conversion regions in the plasma sheet. Due to experimental limitations, investigating the power density $\boldsymbol{E} \cdot \boldsymbol{J}$ from in situ data is difficult. At least four spacecraft are needed to obtain the full current density vector from the curl of the magnetic field. Moreover, the expected power density of one or a few $\mathrm{pW} / \mathrm{m}^{3}$ in many regions of the plasma sheet (Birn and Hesse, 2005; Marghitu et al., 2006) implies electric and magnetic field measurements close to the instru- ments detection limits.

Cluster allows for the first time a systematic examination of energy conversion in the Earth's magnetosphere from in situ data. By investigating the power density $\boldsymbol{E} \cdot \boldsymbol{J}$, loads $(\boldsymbol{E} \cdot \boldsymbol{J}>0)$ and generators $(\boldsymbol{E} \cdot \boldsymbol{J}<0)$ can be identified. To our knowledge, the first experimental investigations of generator regions in the plasma sheet were presented in Marghitu et al. (2006); Hamrin et al. (2006). During a very strong geomagnetic activity a clear generator was identified at the magnetopause flank of the tail (Rosenqvist et al., 2006). The generators were identified as concentrated regions with negative power densities as obtained by Cluster. These regions were labelled Concentrated Generator Regions (CGRs) and they were observed near the Plasma Sheet Boundary Layer (PSBL) at an altitude of about 18 Earth radii. The CGRs were also shown to correlate with auroral electrons observed by the FAST satellite. Based on a manual selection of Energy Conversion Regions (ECR) events, a preliminary statistical investigation of energy conversion in the plasma sheet was presented in Marghitu et al. (2009). The energy conversion was shown to be rather structured, and the traversal of the ECRs (typically in the GSE -z-direction) was fast, of the order of $10 \mathrm{~min}$, which is much shorter than the time needed by Cluster to cross the plasma sheet (several hours). Depending on the sign of the power density, these regions are therefore called Concentrated Generator Regions (CGRs) and Concentrated Load Regions (CLRs), and they are supposed to be distinguished from any possible distributed ECRs which may perhaps extend over much larger regions in time and/or space. The word concentrated refers specifically to the GSE z-direction, but a case study analysis in Marghitu et al. (2006) suggests that the CGRs might as well be concentrated in the other directions, and in time. Marghitu et al. (2006) estimated a lower limit of the CGR extension along the field line to about $1000 \mathrm{~km}$.

In this article we continue the work initiated by Marghitu et al. (2009) by presenting a comprehensive statistical investigation of CLRs and CGRs in the plasma sheet. The investigation is based on more than 80 Cluster plasma sheet crossings (or $660 \mathrm{~h}$ of data) in the summer and fall of 2001 when Cluster was close to apogee (at an altitude of about $15-20 R_{E}$ ). The present investigation is based on an automatic event selection from the Cluster power density data (as opposed to the manual selection in Marghitu et al., 2009). Our present data base allows for extended investigations of the occurrence and location of ECRs in the Earth's plasma sheet observed by Cluster in 2001. In a companion paper, Hamrin et al. (2009) [Scale size and life time of energy conversion regions observed by Cluster in the plasma sheet], we investigate the life time and scale size of the ECRs. Hereafter, we will refer to the companion paper as H09B. In H09B we show that the ECRs indeed are concentrated in space as well and time, and typical scale sizes of CLRs and CGRs are obtained. 


\section{Instrumentation and method}

The four Cluster Spacecraft were launched in 2000 into a polar orbit (inclination $81^{\circ}$ ) with apogee and perigee at $18 R_{E}$ and $3 R_{E}$, respectively. The orbital period is $57 \mathrm{~h}$ and the satellites are spin stabilized with a rotation period of $4 \mathrm{~s}$. Cluster is equipped with a complete set of plasma and field instruments (see Escoubet et al., 2001, and references therein).

\subsection{Current density and electric field}

In this article we investigate energy conversion in the plasma sheet by evaluating the power density, $\boldsymbol{E} \cdot \boldsymbol{J}$, where $\boldsymbol{E}$ is the electric field and $\boldsymbol{J}$ the current density. Clear signatures of $\boldsymbol{E} \cdot \boldsymbol{J}>0$ are identified as Concentrated Load Regions (CLRs), and clear signatures of $\boldsymbol{E} \cdot \boldsymbol{J}<0$ as Concentrated Generator Regions (CGRs).

As discussed in Marghitu et al. (2006), GSE and GSM are the appropriate reference systems to use for calculating the power density. They differ only a few degrees from the DSI (Despun Satellite Inverted) system which is the most convenient choice for the EFW instrument.

The full current density vector used in the calculation of the power density can be derived from simultaneous magnetic field measurements from the four FGM instruments (Balogh et al., 1997) on board the spacecraft by using the curlometer method, $\mathbf{J}=\nabla \times \mathbf{B} / \mu_{0}$ (Robert et al., 1998; Dunlop et al., 2002). The quality of the curlometer estimate is rather sensitive to the size and shape of the Cluster tetrahedron. Current density structures smaller than the characteristic size of Cluster cannot generally be resolved with the curlometer. In our data base of plasma sheet crossings in the summer and fall of 2001, the characteristic size of the Cluster tetrahedron is about $\sim 1500 \mathrm{~km}$ which covers a few ion gyroradii (the average proton gyroradius is about $400 \mathrm{~km}$ ). The planarity and elongation of the tetrahedron (Robert et al., 1998 ) is generally around or below 0.1 for our events. This guarantees a tetrahedron close to equilateral which is optimal for the curlometer method.

Three instruments on board Cluster can be used to obtain the electric field, $\boldsymbol{E}$, needed in the calculation of the power density. The Electric Fields and Waves experiment (EFW) (Gustafsson et al., 1997, 2001) and the Electron Drift Instrument (EDI) (Paschmann et al., 2001) are designed to measure the electric field directly. In addition, the drift of low energy plasma ions as detected by the Cluster Ion Spectrometer (CIS) (Rème et al., 2001) can be used to estimate the electric field on the assumption that the $\boldsymbol{E} \times \boldsymbol{B}$ drift is dominant. The CIS experiment consists of a mass and energy ion spectrometer CODIF (Composition and Distribution Function) and an energy ion spectrometer HIA (Hot Ion Analyzer).

The EDI instrument, which measures the drift of a weak test electron beam and then estimates the electric field, does not operate in the plasma sheet since the magnitude of the magnetic field is too small. Moreover, CIS is not operational on all Cluster spacecraft. CODIF is not operational on $\mathrm{C} 2$ and HIA on $\mathrm{C} 2$ and $\mathrm{C} 4$. EFW on the other hand is operational on all spacecraft. However, since the magnetic field vector generally is too close to the satellite spin plane (the DSI xy-plane) containing the EFW probes, we can only obtain electric field components in that plane. Full electric field vectors are hence not available from EFW. The DSI xcomponent of the EFW electric field can sometimes suffer from an offset of the order of $1 \mathrm{mV} / \mathrm{m}$. To reduce the amount of additional calibration of the EFW data, we only use the DSI y-component of the EFW electric field. To obtain the power density, in this article we mainly use vector electric field data from CODIF and HIA computed as $\boldsymbol{E}=-\boldsymbol{V} \times \boldsymbol{B}$. The DSI $E_{y}$ electric field component from EFW is used only for cross-checking the results obtained by CIS.

Notice that the curlometer current density is based on simultaneous measurements of the magnetic field on board the four Cluster spacecraft. It can therefore be interpreted as an average value $(\langle\boldsymbol{J}\rangle)$ over the Cluster tetrahedron. The electric field should hence be averaged over the tetrahedron volume to obtain consistent estimates of the power density over the space spanned by the Cluster spacecraft. Therefore, whenever appropriate, we use the CIS electric field averaged over all available satellites for evaluating the power density. To obtain the best possible electric field average and to increase the accuracy, both CODIF and HIA are included in the computation of the average value, $\langle\boldsymbol{E}\rangle$. Also other quantities, e.g. the magnetic field, are averaged over the Cluster tetrahedron. However, if not stated otherwise, in the following we simplify the notation by omitting the brackets around $\boldsymbol{E}, \boldsymbol{J}$, and $B$.

It should be noted that there are cases when the Cluster spacecraft appear to be located close to the edge of an ECR. Then one or a few satellites might in fact be positioned outside the ECR. This reduces the ECR signature in the power density averaged over the spacecraft. Assuming that small scale fluctuations in the current density do not dominate the properties of the power density, in such cases it would be more appropriate to calculate the power density by using the electric field measured by a single spacecraft only. In this article, and in the companion paper H09B, we clearly state whenever unaveraged electric fields are used.

\subsection{Automatic selection}

To identify CLRs and CGRs in the Cluster data we use an automatic selection routine which searches for clear concentrated regions with $\boldsymbol{E} \cdot \boldsymbol{J}>0$ and $\boldsymbol{E} \cdot \boldsymbol{J}<0$, respectively. The evaluation of the power density is based on electric field and current density data sampled every $4 \mathrm{~s}$.

A schematic example of a CLR is presented in Fig. 1. The top panel shows the power density measured within the Cluster tetrahedron along the spacecraft path. The CLR is highlighted in yellow in the figure and it is identified as a legible 

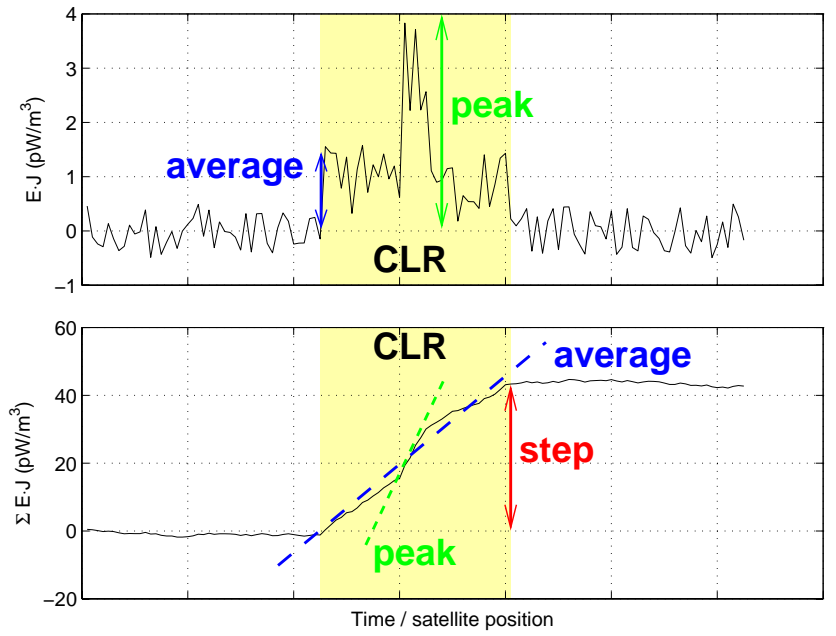

Fig. 1. Schematic CLR (Concentrated Load Region) observed in the power density data. The top panel shows the power density. The bottom panel contains the time integral of the power density along the satellite path. The quantities peak and average correspond to the maximum and average value of the time series of the power density, and hence the maximum and average slope in the integrated power density. The step size measures the increase in the integral. The CLR is highlighted in yellow and it is identified as a clear and concentrated region of $\boldsymbol{E} \cdot \boldsymbol{J}>0$ in the top panel and a positive step in the integrated power density in the bottom panel. A CGR (Concentrated Generator Region) behaves similarly, although $\boldsymbol{E} \cdot \boldsymbol{J}<0$ and the integrated power density instead shows a decrease.

increase in the power density, with $\boldsymbol{E} \cdot \boldsymbol{J}>0$ clearly above the surrounding fluctuations. The peak (maximum) and average value of the power density within the event are indicated in the figure. To reduce the influence of outliers in the data, the median value is used to represent the average. The second panel of Fig. 1 shows the power density integrated along the satellite path. The CLR shows up as a distinct positive step in the integrated power density. The step size of the CLR is defined as the increase in the integrated power density according to the bottom panel in the figure. Note that the average and peak (maximum) values of the power density from first panel evidently correspond to the average and maximum slope of the integrated power density presented in the bottom panel. In the following we will use the quantities peak, average, and step when discussing the strength of the ECRs. The average is computed as the step divided by the 4 times the number of $4 \mathrm{~s}$ samples included in the ECR. Note that the CIS ground data, used to compute the vector electric field, often have a time resolution of 8 or $12 \mathrm{~s}$ (sometimes more). In such cases the data are re-sampled to $4 \mathrm{~s}$.

For a CGR, the picture is similar. However, in this case $\boldsymbol{E} \cdot \boldsymbol{J}<0$ and the integrated power density shows a distinct decrease instead. Moreover, the peak value corresponds to the minimum power density (largest negative value), and hence the largest negative slope in the integral.
The automatic ECR event selection algorithm used in this article is based on three separate steps. In the first step (1. Selection) we identify possible ECR events from the slope of the integral of the power density. Only regions with large enough slopes are kept. However, many of the identified regions from the first step are very small, and they can be located very close to each other, perhaps with some small regions with somewhat noisy data in between. Therefore we need a second step (2. Merging) where CLRs are merged with neighbouring CLRs, and CGRs with neighbouring CGRs.

In the final step (3. Rejection) all ECRs which do not satisfy a set of physical and instrumental requirements are rejected. For example, to ensure that the ions behave collectively within the selected ECRs, the approximate scale size of the ECRs (simply estimated as $\Delta T V_{p l}$, where $\Delta T$ is the time extent of each ECR and $V_{p l}$ is the average plasma flow within the ECR) should be larger than five proton gyroradii. Moreover, the size and shape of the Cluster tetrahedron should be satisfactory and measurements from CODIF, HIA, and EFW should correlate. To ensure that the signatures in $\boldsymbol{E} \cdot \boldsymbol{J}$ are statistically significant, we also require that all ECRs are at least 100 s long, i.e., they are composed of at least 25 data points sampled every $4 \mathrm{~s}$. To be on the safe side, we also reject all events whose power density appear to be very fluctuating and which could be caused by too much noise. It should be noted that small scale fluctuations (smaller than the scale size of the Cluster tetrahedron) in the electric field and current density cannot be correctly resolved in the $\boldsymbol{E} \cdot \boldsymbol{J}$ data. It is impossible to distinguish between fluctuations, e.g., due to random noise and due to small scale fields and currents. By rejecting events with very fluctuating power densities we therefore avoid both noisy data and data on sub-scales.

The individual steps in the automatic selection routine are explained more thoroughly in Appendix A.

The automatic routine has been run on power density data computed both from the electric field averaged over all available spacecraft, but also on the power density obtained from the electric field from single spacecraft. By using nonaveraged electric fields we are able to identify ECRs which may be located close to the edge of the Cluster tetrahedron (cf. the discussion in Sect. 2.2). Data of all selected ECRs have been manually inspected to ensure a successful performance of the automatic selection routine. In this manual examination, a few ECRs have been rejected usually due to noisy data. Also the start and stop time of a few ECRs have been adjusted slightly. However, overall we consider that the automatic selection routine functions satisfactory. Figure 2 shows typical examples of ECRs automatically selected from a few hours of data on 12 August 2001. Regions highlighted in red correspond to CLRs and blue correspond to CGRs. In Fig. 2 we also see a few regions which to the eye look like CLRs (e.g. 16:05 and 17:35 UT) or CGRs (e.g. 15:15 and 22:15 UT). However, these have not been accepted by the automatic routine. For example, possible events around 15:15, 
16:05 and 17:35 UT are rejected due to the appearance of magnetosheath-like signatures in the data (see Appendix A for a discussion on how to automatically distinguish magnetosheath data from plasma sheet data). Possible events observed between about 19:00 and 22:30 UT are also lost from the statistics. In this case the data are too noisy for any events to be kept.

As discussed in Sect. 1, the power densities obtained in the plasma sheet imply electric and magnetic field measurements close to the instruments detection limits. In the case of the electric field, there are three separate instruments which can be used for cross-checking the results. Figure 3 shows average power densities (average slope in the integrated power density) for all selected ECRs. (Cf. Fig. 1 for a definition of the average power density.) As in the rest of this article, red corresponds to CLRs and blue to CGRs. To the left in Fig. 3 the average power density obtained by using the HIA electric field is plotted versus the average power density obtained from CODIF. To the right the average power density from EFW is plotted versus CODIF. Note that only the DSI $y$ contribution, $E_{y} J_{y}$, is considered when cross-checking with EFW. Since EFW data is not available for all selected ECRs, the right plot contains fewer data points. Generally we see that the instruments agree rather well on the power density for the selected ECRs, indicating reliability of the results. The correlation coefficients (using non-log data) are 0.92 for the left plot and 0.86 for the right plot.

To be able to analyze the selected CLRs and CGRs, and to separate their characteristics from the general characteristics of the plasma sheet, we have compared our ECRs with a data base of randomly selected time intervals evenly spread within the plasma sheet data of the summer and fall of 2001. The time extent $\Delta T$ of the random events are chosen from a square distribution between $100 \mathrm{~s}$ and $1000 \mathrm{~s}$. The same number of random events are selected from each Cluster orbit, but only events within the plasma sheet (as identified by the automatic routine, see Section A) are retained. The random events are not allowed to overlap each other. Note that events are rejected afterwards if the CODIF and HIA data do not correlate, but no further physical or instrumental requirements are used in the automatic routine. In the following we will refer to this data base as a random data base. It contains in total 918 time intervals. By examining the sign of the step size in the integrated power density, we can identify 480 random events with load signatures (positive step, $\boldsymbol{E} \cdot \boldsymbol{J}>0$ ) and 438 random events with generator signatures (negative step, $\boldsymbol{E} \cdot \boldsymbol{J}<0)$. In the following, these events will be called random loads (RAND-L) and random generators (RAND-G), respectively. Note that these random events should not be confused with our ordinary data base of CLRs and CGRs which are carefully selected from the plasma sheet data and which satisfy a set of requirements which guarantees their reliability as ECRs. Since the random data base only consists of randomly selected time intervals, there is no guarantee that they correspond to true ECRs. Instead the random data base is merely used as a reference, for capturing the typical behavior of the plasma sheet.

\section{Observations}

From $660 \mathrm{~h}$ of plasma sheet data in the summer and fall of 2001, in total 151 ECRs have been identified. This corresponds to the occurrence of one ECR observed approximately every four hours in the plasma sheet. However, this is probably an underestimate since other relevant energy conversion regions might well exist in the plasma sheet data, but are not included in our statistics since they are not identified by the automatic routine for one reason or another. It should be noted that our ECRs are identified by an automatic routine which only selects those events which satisfy a set of physical and instrumental requirements. The data base hence only contains the clearest ECRs with the most typical power density signatures. Weaker or more atypical power density signatures are not included in our statistics.

In the hour-DOY (Day Of Year) plot in Fig. 4, the grey lines represent the coverage of the Cluster data used in this investigation. Note that only data from the plasma sheet, as identified by the automatic selection routine, are included. This explains the somewhat scattered gray points in some regions of the plot. In the same figure, all identified CLRs and CGRs are also indicated by the red and blue lines, respectively. From Fig. 4 we conclude that the identified ECRs originate from several separate orbits. Even though it is outside the scope of the present investigation to include information of magnetospheric activity indices such as $\mathrm{AE}$ and $\mathrm{Kp}$ into the data base, one might expect a positive correlation between those regions in Fig. 4 where the ECRs are observed and intervals of higher magnetospheric activity. Magnetic activity and their relation to the ECRs will be the issue for future investigations.

From Fig. 4 we also note that the time extent $\Delta T$ of the observed ECRs in many cases are rather short. As we discuss further in H09B, we believe that an ECR life time of about 1-10 min can be obtained from measurements of $\Delta T$.

We find that 116 (35) out of the identified events are CLRs (CGRs). Hence, almost $80 \%$ of the ECRs have been identified as CLRs. In the Cluster plasma sheet data, one CLR is observed approximately every six hours while one CGR is observed only every $22 \mathrm{~h}$. Thus the plasma sheet seems to behave more often as a load. In this article we aim to reveal the structure of this large scale plasma sheet load.

To estimate the strength of the plasma sheet load, we evaluate the typical power density of the automatically selected ECRs, as well as the typical power density of the random selected time intervals from the plasma sheet (cf. Sect. 2.2 for a discussion of the random data base). The result is shown in Fig. 5. The three panels show the ECR strength measured by the step, peak, and average power density as defined in Fig. 1 (see also Sect. 2.2). Typical strengths are both computed as 


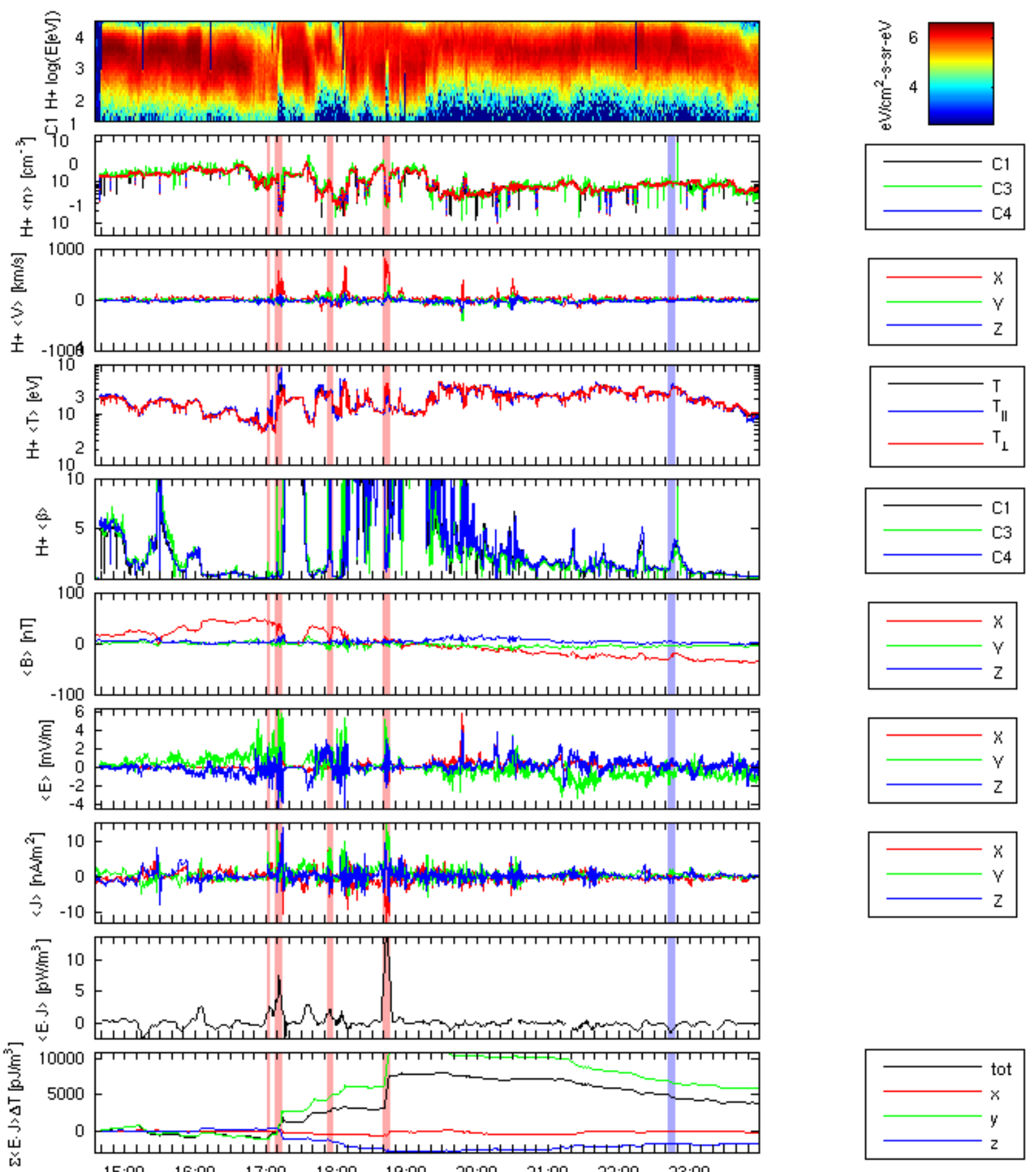

Fig. 2. ECRs automatically selected from a few hours of CODIF and FGM Cluster data. Highlighted regions correspond to CLRs (red) and CGRs (blue), respectively. The panels from top to bottom show: (a) CODIF proton energy spectrogram for C1. (b) Proton density obtained by C1, C3, and C4. (c) Plasma flow in GSE $x, y$, and $z$ computed as an average over C1, C3, and C4. (d) Average, parallel and perpendicular proton temperature computed as an average over $\mathrm{C} 1, \mathrm{C} 3$, and $\mathrm{C} 4$. (e) The plasma beta obtained by $\mathrm{C} 1, \mathrm{C} 3$, and $\mathrm{C} 4$. (f) The magnetic field in GSE $x, y$, and $z$ computed as an average over C1, C3, and C4. (g) The GSE $x, y$, and $z$ components of the electric field average over C1, C3, and C4. (h) The GSE $x, y$, and $z$ components of the current density average over C1, C3, and C4. (i) The power density $\boldsymbol{E} \cdot \boldsymbol{J}$ along the spacecraft orbit (the power density has been smoothed with a $5.5 \mathrm{~min}$ running average to increase the visibility). (j) The time integral of the power density along the satellite path (no running average is used). Red, green and blue show contributions from the GSE $E_{x} J_{x}, E_{y} J_{y}$, and $E_{z} J_{z}$ components. Black lines correspond to the full product $\boldsymbol{E} \cdot \boldsymbol{J}$. To avoid any misinterpretations in the figure, note that we have kept the brackets around the quantities averaged over the spacecraft tetrahedron. 

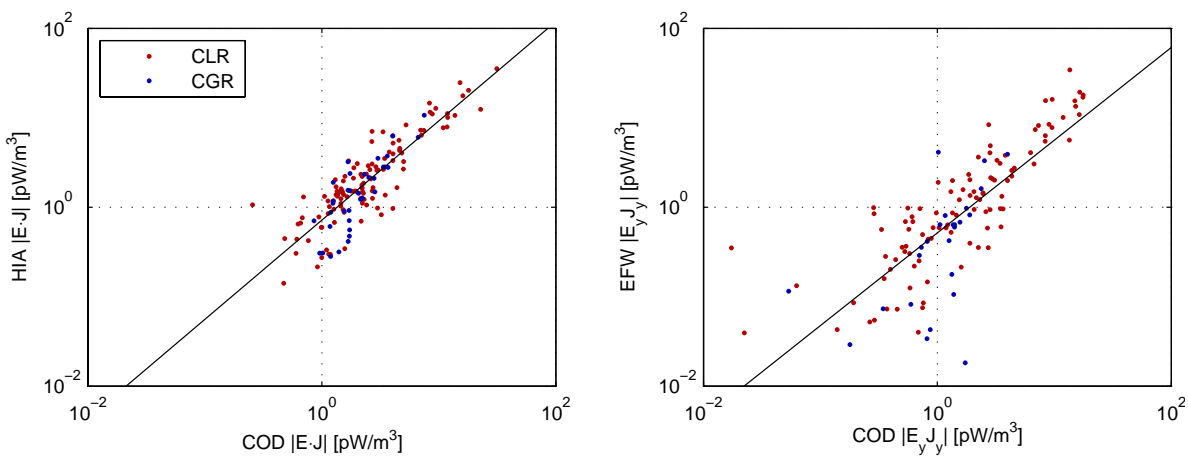

Fig. 3. Cross-check of the power density obtained for the selected ECRs by using electric field estimates from CODIF, HIA and EFW (only the DSI y-component is used for cross-checking with EFW). Straight lines (on the logarithmic scale) have been fitted to the data. Left: $|\boldsymbol{E} \cdot \boldsymbol{J}|$ for HIA versus CODIF. Right: DSI $\left|E_{y} J_{y}\right|$ from EFW versus CODIF. Red and blue correspond to CLRs and CGRs, respectively. Only the absolute value of the power density is plotted. Note that the same scalings of the axes are used. Correlation coefficients for the non-log data are 0.92 for the left plot and 0.86 for the right plot. We see that the correlation between the instruments is generally good.

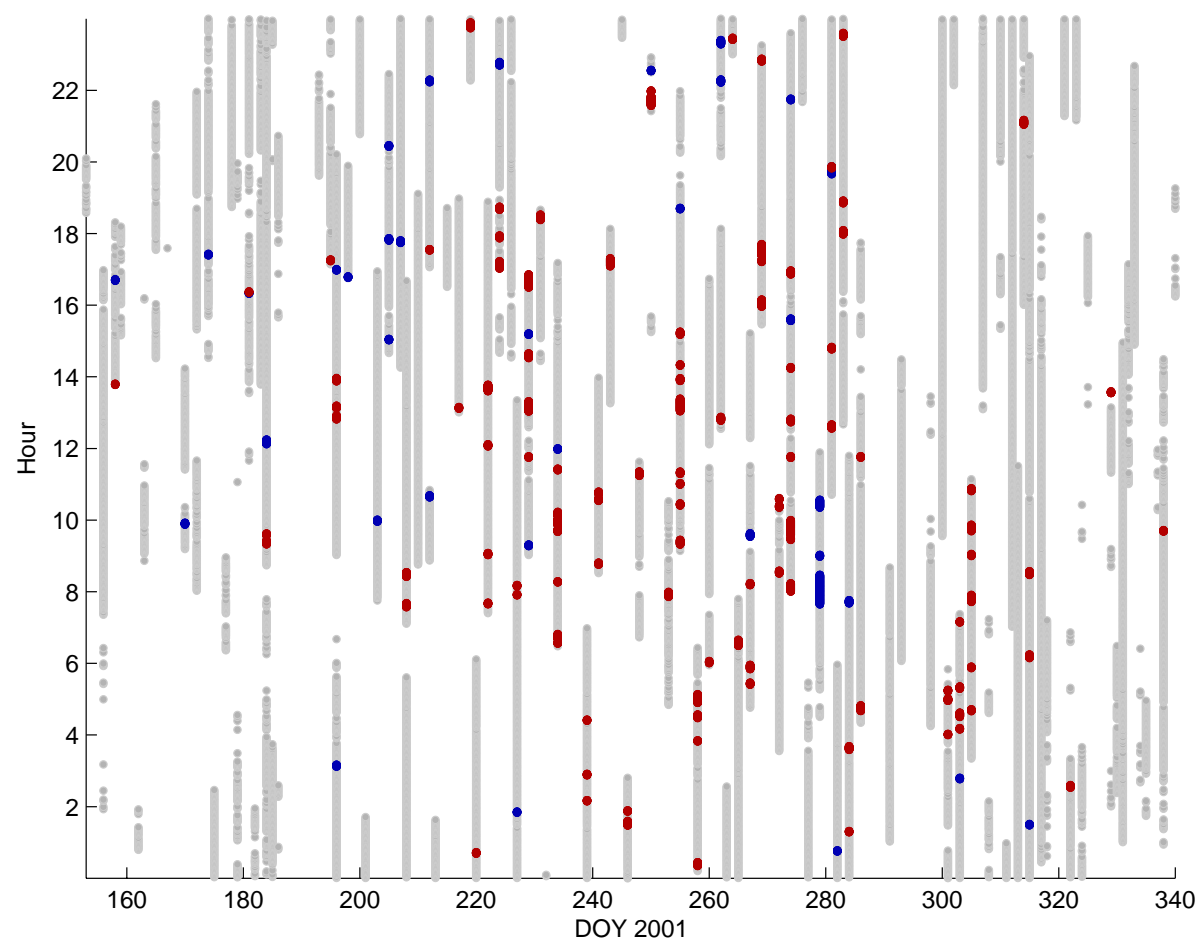

Fig. 4. Hour-DOY (Day Of Year) plot of the coverage of the Cluster plasma sheet data used in this investigation (grey lines). The red and blue dots indicate the CLRs and CGRs, respectively, observed by the mission. We note that the observed ECRs originate from several separate satellite orbits. Moreover, the time extent of many ECRs are rather short, of the order of about 1-10 min (cf. H09B).

mean values of available events (coloured bars) as well as median values (white bars). For the coloured bars, the color coding is the same as for the rest of the article: Red and blue correspond to CLRs and CGRs, respectively, while light red and light blue signify RAND-Ls and RAND-Gs, i.e., the random time intervals selected within the plasma sheet.
For all three panels, and independently of averaging method (mean or median), we see that CLRs are stronger than CGRs, and RAND-Ls are stronger than RAND-Gs. Hence, the plasma sheet behaves on average as a load, as expected from the large scale dawn-to-dusk orientation of the electric field and current. Nonetheless, at certain times smaller scale regions may well have a generator character. 

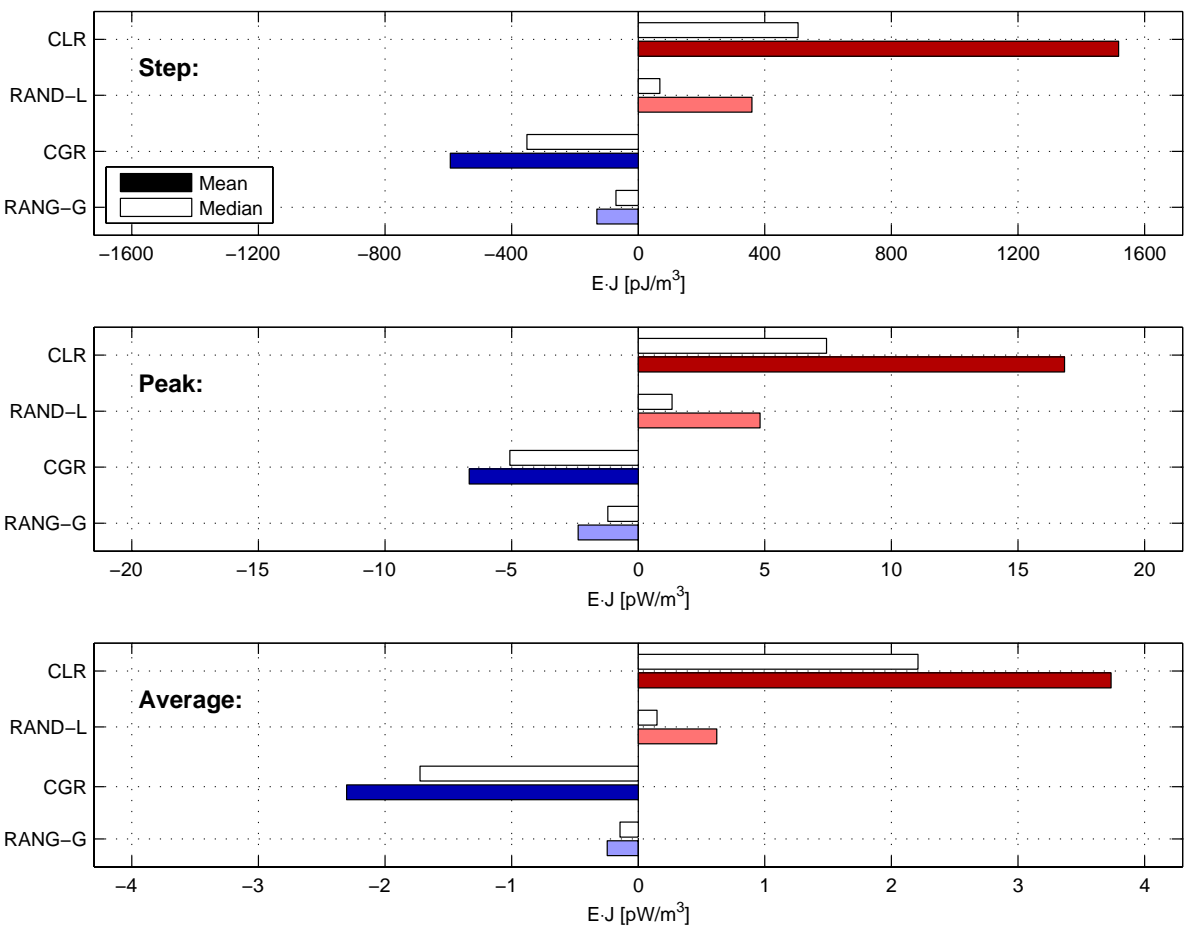

Fig. 5. Strength of the load and generator regions in the plasma sheet data from the summer and fall of 2001. The three panels show the step, peak and average strengths (cf. Fig. 1). Red and light red correspond to CLRs and RAND-Ls while blue and light blue correspond to CGRs and RAND-Gs. Note the different scalings of the $\mathrm{x}$ axes. On the average we see that the plasma sheet near $18 R_{E}$ behaves as a load although it hosts both CLRs and CGRs.

The random data base should be used as a reference data set. It reflects the overall behaviour of the plasma sheet at an altitude of about $18 R_{E}$. There are no requirements on the random events other than that CODIF and HIA data should correlate. Since the RAND events are randomly selected from all available data, this implies that some ECRs might well also be included in the random data base. However, the ECRs referred to in this article correspond to concentrated regions of energy conversion, stronger and clearly visible to the eye (and to the automatic selection routine) from the surrounding data. This explains why the typical strength of RAND-Ls and RAND-Gs are considerably weaker than for CLRs and CGRs.

Our results show that there are slightly more RAND-Ls than RAND-Gs (480 RAND-Ls and 438 RAND-Gs). Moreover, the RAND-Ls are stronger than the RAND-Gs. This is again consistent with the plasma sheet behaving, on the average, as a load.

As discussed before, there are various ways to characterize the typical strength of the ECRs (see Fig. 1), for example the step size of the integrated power density, the peak value of the power density, and its average. As seen from Fig. 5, estimating the typical ECR strength by using the step value, we obtain larger values than if we use the peak or average. This is due to a dependence on the time extent $\Delta T$ of the
ECRs. The step value does not compensate for large $\Delta T$, resulting in larger steps for longer ECRs. Also the peak estimate can show a (weak) dependence on $\Delta T$, since there is a greater probability of a long time series to show large fluctuations. Moreover, the peak value is also more sensitive to instrumental errors and accidental outliers that may influence the result. For these reasons, step and peak are generally less suitable for a detailed analysis of the strength of the ECRs. In this article we will therefore use the average whenever the ECR strength is considered. However, from Fig. 5 we see that all three measures (step, peak or average) imply the same thing: the loads are stronger than the generators and the plasma sheet behaves in general as a load. This is consistent with results from Marghitu et al. (2009).

From Fig. 5 we also note that the coloured bars (mean values) generally are larger than corresponding white bars (median values). Apparently there exist strong ECRs which increase the mean value as compared to the median. The spread of the ECR strength is also reflected by the standard deviations (not shown) which are largest for CLRs and RAND-Ls, where outliers in the data are stronger. The largest relative spread is observed in RAND-Ls, where the weight of the strong outliers is larger than for the (more constrained) CLRs. Comparing the ECR strength on a logarithmic scale (not shown) we see that the ECRs are generally 

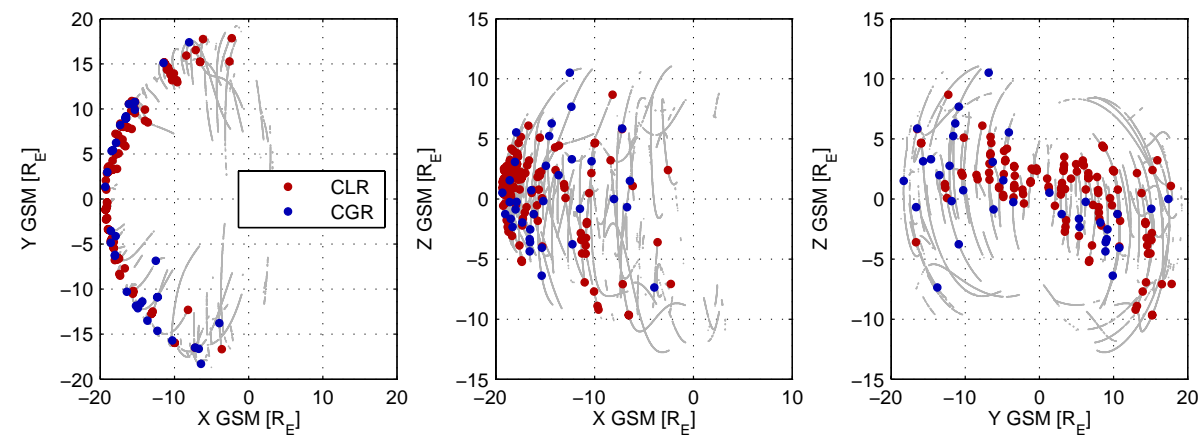

Fig. 6. The localization of CLRs (red) and CGRs (blue) in GSM $x y z$ space. The grey lines indicates the Cluster sampling in the plasma sheet during the summer and fall of 2001. The right plot shows that CLRs generally prefer locations deeper into the plasma sheet and closer to the neutral sheet.

logarithmically distributed, resulting in higher mean values as compared to the median values.

Based on the random reference data base, we next compute the mean value of the average energy conversion within the plasma sheet (independently of the classification into RAND-Ls and RAND-Gs). This results in a typical energy conversion of about $0.2 \mathrm{pW} / \mathrm{m}^{3}$ of the plasma sheet in general. Hence, again the plasma sheet behaves as a load. Notice that this estimate is based on the assumption that all random data are reliable and not affected too much by noise (since we require a sufficient correlation between results from CODIF and HIA, this risk is of course reduced). Calculating the similar mean value, but only for the selected CLRs and CGRs, we obtain a energy conversion of about $2.3 \mathrm{pW} / \mathrm{m}^{3}$. However, this is probably an overestimate. The automatically selected ECRs are supposed to be the most prominent ECRs within the plasma sheet. One might expect that less pronounced ECRs could also exist in the plasma sheet although they cannot be identified by the present selection routine. It is reasonable to expect that the average ECR strength in the plasma sheet at an altitude of about $18 R_{E}$ is somewhere between these two values above, i.e. between $0.2 \mathrm{pW} / \mathrm{m}^{3}$ and $2.3 \mathrm{pW} / \mathrm{m}^{3}$, i.e., of the order of $\sim 1 \mathrm{pW} / \mathrm{m}^{3}$, which is consistent with previous estimates in Marghitu et al. (2006). Rough calculations show that this average power density level (of $\sim 1 \mathrm{pW} / \mathrm{m}^{3}$ ) appears to be consistent with the solar wind input

To determine the ECR location with respect to the neutral sheet, in Fig. 6 we plot the automatically selected CLRs (red) and CGRs (blue) in the GSM $x y, x z$, and $y z$ planes. The light grey lines show where in the plasma sheet the Cluster satellites have been probing during the summer and fall of 2001. We note that the Cluster plasma sheet crossings of 2001 spans over altitudes of approximately $15-20 R_{E}$, say, about $18 R_{E}$. From the right plot we see that CLRs generally appear to exist closer to the central plasma sheet, while CGRs prefer locations further out. According to the simulations of Birn and Hesse (2005), generator regions generally exist close to the plasma sheet boundary layer (PSBL). Comparing the location of the CGRs with the outer bordering grey lines in the GSM $y z$ plot we may conclude that the CGRs prefer regions closer to the PSBL which would be consistent with Birn and Hesse (2005).

The outermost grey lines in Fig. 6, for example $y>0$ and $z>5 R_{E}$, correspond to the statistical boundary region between the plasma sheet and the magnetosheath or the lobes. Our automatic selection routine is able to identify these boundaries to a good extent (see Appendix A) and rejects all Cluster measurements which are outside the boundaries. Hence, there are significantly less Cluster data available along those outermost grey lines (not visible from the figure). This is the reason why very few ECRs are identified there. The ECRs located in the boundary layers close to the magnetopause will be examined in a forthcoming paper.

The general motion and the thinning and expansion of the plasma sheet with the substorm cycle prevents any definite conclusions about the ECRs location from the GSM $x y z$ plots. However, a visual inspection of all 35 identified CGRs used in this investigation confirms that a majority of these are indeed located toward the PSBL.

Further information on the location of the ECRs with respect to the central plasma sheet can be obtained by analyzing the magnetic field. Previous investigations (Marghitu et al., 2009) have not included a quantitative evaluation of the variation of the magnetic field. However, the present data base offers better statistics for such an investigation to be possible. Hence, for each ECR in the data base we have calculated the median value of the $B_{x}$ magnetic field component. Note that the magnetic field is averaged over the Cluster tetrahedron. However, to simplify the notation, as usual we omit the brackets in $\left\langle B_{x}\right\rangle$ and denote the median value of the averaged magnetic field simply as $B_{x}$.

Near the neutral sheet, $B_{x}$ should be close to zero. The top panel of Fig. 7 shows a $B_{x}$ histogram. Red corresponds to CLRs and blue to CGRs. The error bars indicate the uncertainty due to limited statistics. Measurement errors are not 

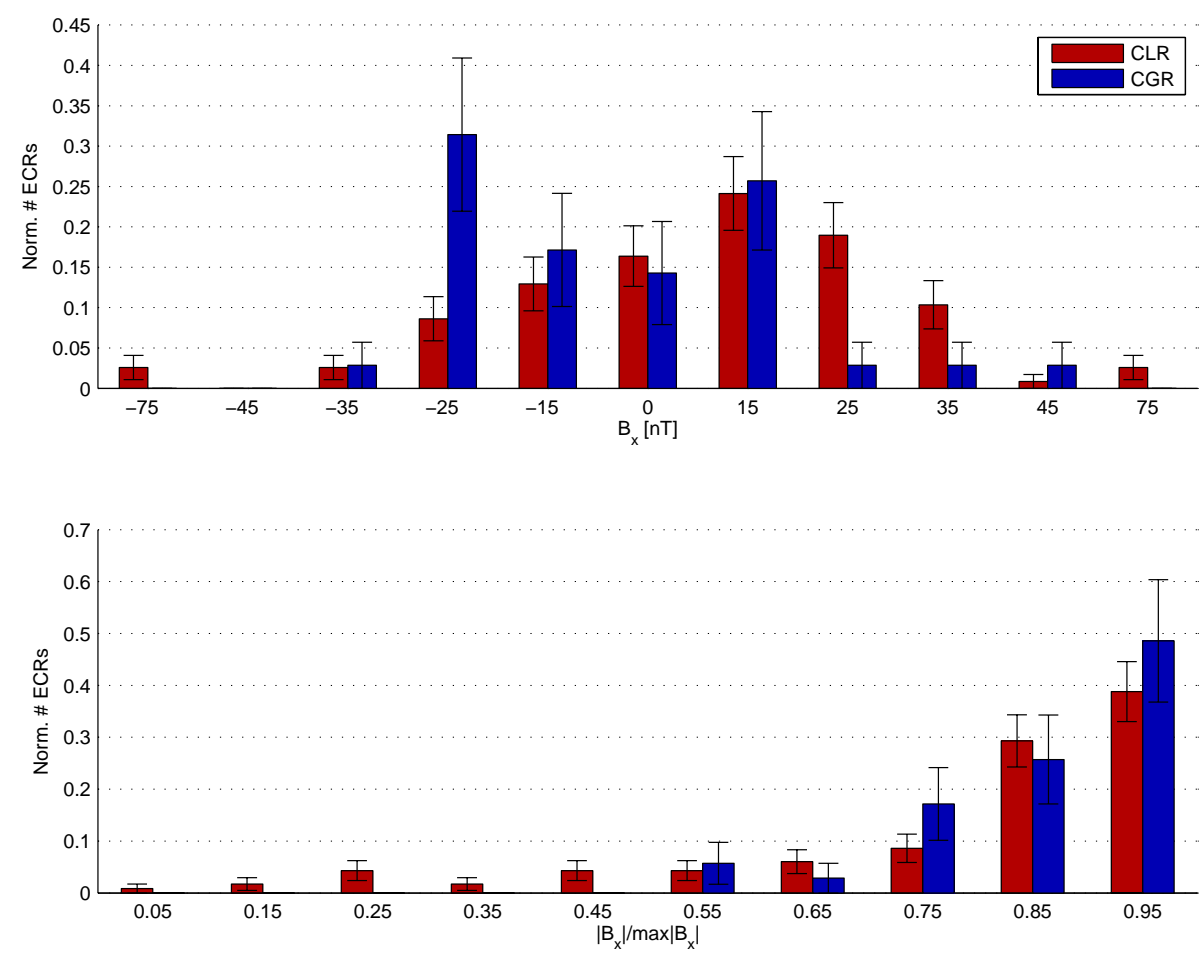

Fig. 7. The median value of the Earthward magnetic field, $B_{x}$, within an ECR and the ratio $\left|B_{x}\right| / \max \left|B_{x}\right|$ can be used for investigating the closeness to the neutral sheet. As for the previous figures, red correspond to CLRs and blue to CGRs. The error bars indicate the uncertainty due to limited statistics. The plots are normalized so that the sum of all red bars is equal to one, and similarly for the blue bars. The ratio $\left|B_{x}\right| / \max \left|B_{x}\right|$ is used for estimating the variation and smallness of $B_{x}$ within the ECR. Small values correspond to larger variations in $B_{x}$ with possible excursions toward $B_{x}$ close to zero. Larger values of $\left|B_{x}\right| / \max \left|B_{x}\right|$, on the other hand, indicate regions with smaller variations and where the median value of $B_{x}$ is rather close to the maximum value. The top panel shows that there is a tendency for CLRs to prefer smaller values of $B_{x}$, with a peak around $15 \mathrm{nT}$, while the CGRs show a double peak, at about $-25 \mathrm{nT}$ and $15 \mathrm{nT}$. Although the evidence is not very strong, possibly because of limited statistics, the CLRs appear to be located closer to the neutral sheet than the CGRs. In the bottom panel the accumulation of CGRs toward larger values of $\left|B_{x}\right| / \max \left|B_{x}\right|$ is slightly more pronounced, supporting the same conclusion.

included in the error bars. The plots are normalized so that the sum of all red bars is equal to one, and similarly for the blue bars. We see that CLRs generally prefer smaller values of $B_{x}$ than CGRs. This is consistent with a location of CLRs closer to the neutral sheet and CGRs away from the neutral sheet, i.e., closer to the PSBL. Note that the ECRs are asymmetrically distributed around $B_{x}=0$, especially for CLRs which dominate around $15 \mathrm{nT}$. This asymmetry might be due to the asymmetric sampling of the plasma sheet as shown by the grey lines in Fig. 6 .

Using $B_{x}$ as a proxy for the location of ECRs close to or away from the neutral sheet of course also suffers from problems with the variations of the plasma sheet, such as thinning and expansion. A value of $B_{x}$ of, say, $20 \mathrm{nT}$, might correspond to a location close to the lobe in one Cluster orbit and deep inside the plasma sheet in another. To avoid this problem, we compare the median value of the Earthward directed magnetic field component $\left(B_{x}\right)$ with the maximum value of the magnitude of the same magnetic field compo- nent $\left(\max \left|B_{x}\right|\right)$ within each ECR. The result is shown as a histogram in the bottom panel of Fig. 7.

In Fig. 7, large values of the ratio $B_{x} / \max \left|B_{x}\right|$, i.e. ratios close to one, correspond to small variations in the magnetic field during the ECR crossings. Small values of $B_{x} / \max \left|B_{x}\right|$, on the other hand, indicates larger variation in the magnetic field, and a small value of the median $B_{x}$ as compared to the maximum one. Small values of $B_{x} / \max \left|B_{x}\right|$ would correspond to regions closer to the neutral sheet, and larger values to regions closer to the PSBL. From Fig. 7 we note that there is a tendency in the location of ECRs with CGRs preferring locations toward the PSBL, and CLRs toward the PSBL. Of course, noise and other fluctuation may complicate the interpretation of $B_{x} / \max \left|B_{x}\right|$. Moreover, the interpretation is also complicated by the fact that that the data in Fig. 7 are averaged over a wide range of local times and GSM $x$ positions (both close to and further from possible reconnection sites). 

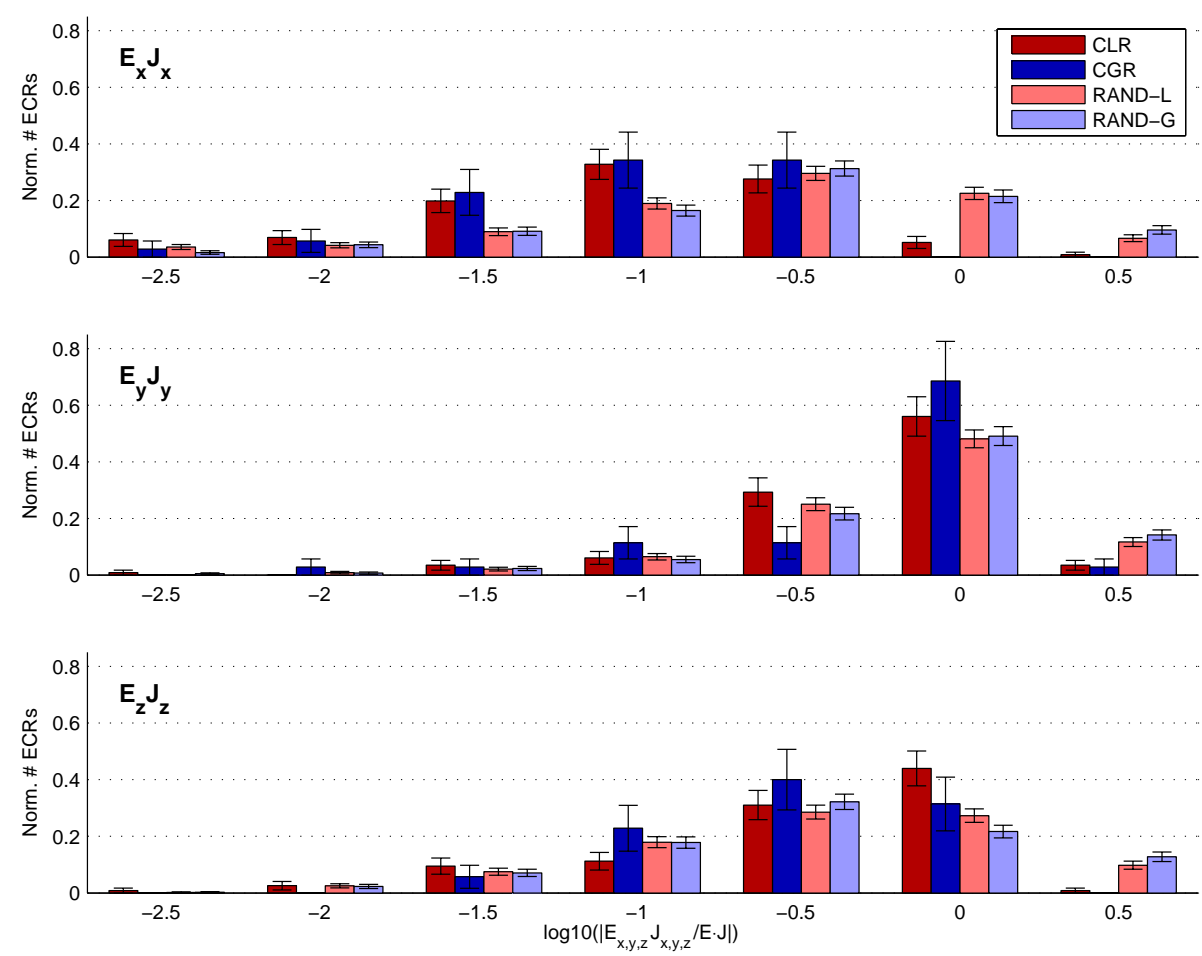

Fig. 8. Histograms of the contribution from various direction to the total power density, $\left|E_{x} J_{x} / \boldsymbol{E} \cdot \boldsymbol{J}\right|,\left|E_{y} J_{y} / \boldsymbol{E} \cdot \boldsymbol{J}\right|$, and $\left|E_{z} J_{z} / \boldsymbol{E} \cdot \boldsymbol{J}\right|$, respectively. The DSI coordinate system has been used for the different electric field and current density components. Red and blue correspond to CLRs and CGRs, respectively, while light red and light blue to RAND-Ls and RAND-Gs. We see that most events have $\left|E_{y} J_{y} / \boldsymbol{E} \cdot \boldsymbol{J}\right| \sim 1$, i.e., $E_{y} J_{y}$ dominates the power density both for CLRs and CGRs (and also for the RAND data).

It is well known that the plasma sheet on the average behaves as a load due to the large scale dawn-dusk electric field and cross-tail current, generally in the GSE y-direction. The dominance of the y-direction can be verified from our data base. In Fig. 8 we present the contribution of $E_{x} J_{x}, E_{y} J_{y}$, and $E_{z} J_{z}$ to the total power density. The error bars indicate the uncertainty due to limited statistics (measurement errors are not included). The median value of $E_{x} J_{x}, E_{y} J_{y}$, and $E_{z} J_{z}$ within each ECR has been computed and the DSI coordinate system has been used for this purpose. This system is optimal for the EFW instrument, and cross-checking between EFW and CIS has been made whenever possible, i.e., whenever EFW data have been available. Note that DSI only differs a few degrees from GSE and GSM. As usual, red and blue correspond to CLRs and CGRs, while light red and light blue correspond to RAND-Ls and RAND-Gs. The number of events are normalized so that the sum the CLR bars is equal to one, and similarly for CGRs, RAND-Ls and RAND-Gs.

We clearly see that the DSI $y$ contribution dominates for both CLRs and CGRs, i.e., indicating the importance of the cross-tail direction for the plasma sheet energy conversion. This is also consistent with the event studies of Marghitu et al. (2006) and Hamrin et al. (2006). Moreover, the z-direction occasionally contributes significantly to the total power density, while the $E_{x} J_{x}$ contribution generally is the smallest one, with many CLRs and CGRs showing $\left|E_{x} J_{x} / \boldsymbol{E} \cdot \boldsymbol{J}\right| \sim 0.1$. Note that $x$ is not necessarily magnetically field-aligned as was the case in Marghitu et al. (2006) and Hamrin et al. (2006). A ratio $\left|E_{x y z} J_{x y z} / \boldsymbol{E} \cdot \boldsymbol{J}\right|$ larger than one in Fig. 8 implies that there exist contributions from other directions which compensate.

From Fig. 8 we also see that the RAND data are dominated by $E_{y} J_{y}$, and to some extent by $E_{z} J_{z}$. This is not unexpected since the randomly selected time intervals provide the reference data base which is supposed to give an overall picture of the plasma sheet.

Further investigating the details of $E_{y} J_{y}$, we have computed the median value of the DSI electric field and current density within each ECR. Figure 9 shows histograms of the result. The colour coding is the same as before. From the top and bottom panels we see that the $E_{y}>0$ and $J_{y}>0$ generally dominates for the CLRs. This again confirms the importance of the global dawn-dusk electric field and cross-tail current for the average load behaviour of the plasma sheet. For CGRs the picture is more ambiguous and a few different combinations of $\boldsymbol{E}$ and $\boldsymbol{J}$ components are possible. The prevalence of the $y$ direction seems to be weaker for the CGRs, possibly related to a higher fluctuation level near the PSBL where 

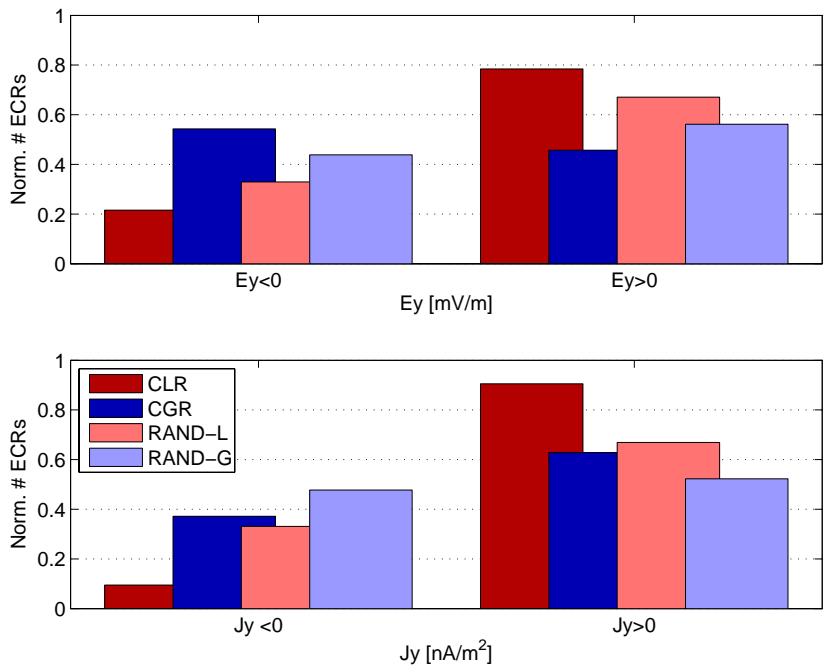

Fig. 9. Histograms of the DSI $y$ electric field and current density. For loads (CLRs and RAND-Ls), we see the expected dominance of $E_{y}>0$ and $J_{y}>0$, while the results for generators (CGRs and RAND-Gs) are less clear.

many CGRs are found. For those CGRs with $E_{y} J_{y}<0$ we see a significant dominance of $E_{y}<0$ and $J_{y}>0$, consistent with Marghitu et al. (2006) and Hamrin et al. (2006). Only in 4 cases out of 35 identified CGRs we find $E_{y}>0$ and $J_{y}<0$. The other CGRs with $E_{y}>0$ hence have $J_{y}>0$, i.e., $E_{y} J_{y}>0$. Therefore, in those cases the total power density $\boldsymbol{E} \cdot \boldsymbol{J}<0$ is caused by contributions from the $z$ and occasionally the $\mathrm{x}$ direction, consistent with a more complicated 3-D nature of the CGRs.

Also in the random reference data base we see a domination of $E_{y}>0$ and $J_{y}>0$ for the RAND-Ls, confirming the expected and overall dependence of the global dawn-dusk electric field and cross-tail current in the plasma sheet. For RAND-Gs, no direct conclusions are possible. However, noise and other fluctuations in the random data could perhaps make the signatures somewhat less clear than for CLRs and CGRs. Note also that contributions from other directions, mainly $E_{z} J_{z}$, can complicate the interpretation of this figure both for CLRs and CGRs, but also for the RAND data.

Figure 10 contains similar information as Fig. 9 but for the DSI $E_{z} J_{z}$ contribution which occasionally dominates the power density. For the $z$ component we see a dominance of $E_{z}>0$ and $J_{z}>0$ for CLRs, and of $E_{z}<0$ and $J_{z}>0$ for CGRs. We do not have an explanation for this asymmetry.

\section{Discussion}

By examining the power density $\boldsymbol{E} \cdot \boldsymbol{J}$, we have investigated the localized energy conversion in the plasma sheet as observed by Cluster in the summer and fall of 2001, when the satellites probed altitudes of about $18 R_{E}$. From $660 \mathrm{~h}$ of data
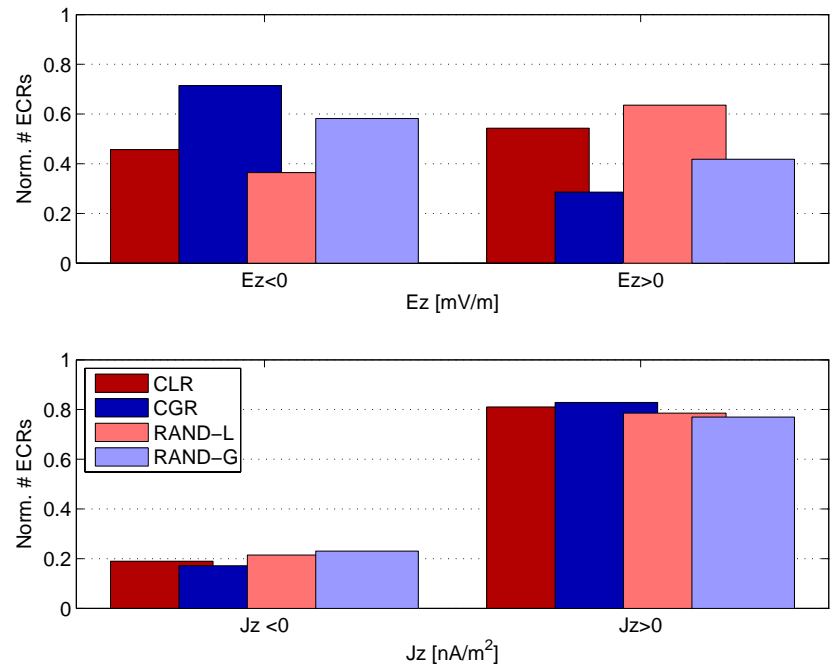

Fig. 10. Histograms of the DSI $z$ electric field and current density.

we have observed 151 concentrated ECRs, of which 116 are identified as CLRs, and 35 as CGRs. The data have been compared with a set of randomly selected time intervals from the plasma sheet during the summer and fall of 2001. The random data have been used as a reference data base portraying the overall behaviour of the plasma sheet.

The data presented in this article support the overall notion that the plasma sheet, on the average, behaves as a load. We note that more than three times as many CLRs than CGRs are identified in the plasma sheet, and that the CLRs also appear to be considerably stronger than the CGRs (cf. Fig. 5). Moreover, we get similar results from the random reference data base (there are slightly more RAND-Ls than RAND-Gs and the RAND-Ls are also stronger).

Characterizing the strength of an ECR is not totally uncomplicated. In this article we have mentioned three possible ways of estimating the ECR strength, the step, peak, and average values as shown in Fig. 1. As discussed in H09B, CLRs tend to extend over longer time intervals, hence increasing the step size for CLRs. Therefore, the step value becomes unsuitable for designating the ECR strength, especially for comparison between CLRs and CGRs. The peak value is particularly sensitive to measurement errors since it corresponds to the largest positive (for CLRs) or negative (for CGRs) value of the power density within the ECR. Any outlier in the data hence has a big impact on the resulting measure. Moreover, the risk for large random fluctuations and outliers are higher for ECRs which extends over larger times. Hence, neither the peak value is good for comparing ECR strengths between CLRs and CGRs.

We find that the average power density (corresponding to the average slope of the power density integrated along the satellite path) in many cases is suitable for characterizing the ECR strength. It is not directly dependent on the time 
extent of the ECRs, and it is rather insensitive to measurement errors and outliers in the data. Therefore, the average value should in general be used for estimating the strength of ECRs.

Investigating the details of the random reference data base as presented in Fig. 5, we notice that the median value (white bars) of the energy conversion is roughly equal for RANDLs and RAND-Gs, independently of the measure of strength (step, peak, or average). Even though our general results confirm the overall load behaviour of the plasma sheet, it does not look trivial that a plasma domain known to behave as a load, hosts both loads and generators of almost equal median intensity. The median step and average values are almost identical for RAND-Ls and RAND-Gs, while the median peak value is slightly larger for RAND-Ls than for RANDGs (not visible from Fig. 5). The load character of the plasma sheet appears to be related to both the prevalence of the CLRs over CGRs, and to the outliers in the ECR and RAND distributions, indicated by the differences between the mean and the median. On the other hand, the intensity of the energy conversion seems to be comparable for load and generator regions, as shown by the median values in Fig 5 .

Looking on the mean value (colored bars), however, we get the expected result of loads being clearly stronger than generators, even in the reference data. Of course, as opposed to the median, the mean is more sensitive to occasional extreme values and outliers. Hence, one might be enticed to believe that the load signature of the plasma sheet, as observed in the random reference data by computing the mean strength, is merely due to measurement errors. However, from the original data base of genuine CLRs and CGRs, we clearly see that the load character is evident from both the median and mean values. The load character of the plasma sheet should be visible in the random data as well as in the original data of selected ECRs, even though the signatures in the random data most likely would be weaker. Since there is a weak tendency of the peak value being larger for RAND-Ls than for RAND-Gs, one might speculate that there in fact exists a small tendency of the RAND-Ls indeed being stronger than RAND-Gs when measured by the peak value.

In this article we have shown that CLRs tend to prefer locations closer to the neutral sheet, while CGRs appear to be located further out in the plasma sheet, possibly in or near the PSBL (cf. Figs. 6 and 7), where fluctuations of the electric field and current density are more significant (Marghitu et al., 2009). This is consistent with the results from largescale resistive MHD simulations of energy conversion and transport in the magnetotail (Birn and Hesse, 2005, in particular Fig. 7a).

Further manual inspection of the available Cluster plasma sheet data from the summer and fall of 2001 indicates that there exist more signatures of interest in the power density data than the concentrated ECRs (CLRs and CGRs) discussed in this article. There seems to exist structures which show the same sign of the power densities over more ex- tended regions in space. Such regions could be labelled distributed loads and distributed generator regions, respectively, depending on the sign of the power density. However, sometimes the extended power density signals are rather weak. To be able to judge whether or not these signatures correspond to real distributed ECRs, or if they are just artefacts due to instrumental errors and random fluctuations, more extensive investigations are needed. Although not explicitly addressed, distributed energy conversion might be related to the RAND data base. This relationship will be subject for future work. In the present investigation we have only focused on the clearest ECRs signatures in the power density data. Distributed ECRs are therefore not included in our statistics.

To our knowledge, the full electric field (all three components) in the magnetotail has rarely been studied in the literature. Rostoker and Boström (1976) investigated a MHD generator mechanism for driving the gross Birkeland current system. Mapping the ionospheric electric field into the tail region, they developed a projection of the magnetospheric electric field in the yz-plane. Specifically away from the nonmidnight meridian in the tail, Rostoker and Boström (1976) reported significant electric fields in the $+\mathrm{z}$ - or $-\mathrm{z}$-direction. Note that the current system considered exists at large scales, possibly driven by a large scale MHD generator. In this article, on the other hand, we focus on smaller scale structures. Only concentrated load and generator regions, i.e. only CLRs and CGRs, clearly visible from the surrounding power density data, are included in the study. More distributed loads and generators, such as those discussed in the previous paragraph, are not considered in the present article. As discussed in Marghitu et al. (2006), observed CGRs appear to be dynamic in nature and they prove to have a rather complicated 3-D wavy structure. The finite life time of the ECRs is further discussed in H09B. Consequently, we cannot make a direct comparison between our rather dynamic ECRs and the large scale electric fields in the yz-plane as reported by Rostoker and Boström (1976). However, from Figs. 8 and 10 in the present article, we note that there exist considerable contributions from $E_{z} J_{z}$ to the CGRs as well as to the CLRs. Moreover, Marghitu et al. (2006) and Hamrin et al. (2006) showed evidence of a few CGRs being related to processes in a larger scale auroral system. The CGRs were observed to be correlated with auroral activity, and they suggested that at least some of the electromagnetic energy generated is carried away by Alfvén waves and dissipated in the ionosphere. It is possible to argue that several CGRs, distributed in space, could provide a significant fraction of the energy required by the auroral activity near the polar cap boundary. However, we cannot conclude that these CGRs constitute parts of a large scale and rather stationary Birkeland current system.

It is interesting to investigate whether it is variations in the electric field or in the current density, or both, which govern the existence of an ECR. Does an ECR appear due to a change in the electric field or in the current density? By visual inspection of all 151 included ECRs, in general we note 
that an ECR appears when there is a change both in the current density and the electric field signatures. Note that there also exist some cases when the change in the current density is the quantity dominating over the electric field, and vice versa. However, there is no statistical significant observation that variations in one of the quantities (the electric field or the current density) should be more important than the other one for the resulting power density signature.

\section{Summary and conclusions}

In this article we have presented a comprehensive statistical investigation of localized energy conversion in the plasma sheet. As compared to previous statistical investigations of the plasma sheet (Marghitu et al., 2009), the present study is based on an automatic instead event of a manual event selection. This results in a more objective and consistent event selection, and data availability which allows more quantitative investigations of signatures in the power density data, as well as in other available data such as the magnetic field data. Moreover, the present investigation is based on a larger amounts of data than the previous one.

However, it should be noted that we do not expect to identify all possible CLRs and CGRs existing in the region of the plasma sheet probed by Cluster in the summer and fall of 2001. To obtain a reliable data base of ECRs, the selection criteria for the automatic routine are carefully chosen. Only the clearest ECR signatures are hence included in the data base. This naturally affects for example the estimate of the occurrence frequency of events, making it an underestimate.

In total, we have examined $660 \mathrm{~h}$ of Cluster plasma sheet data from the summer and fall of 2001 when Cluster was close to apogee at an altitude of $\sim 18 R_{E}$. By analyzing the local power density, $\boldsymbol{E} \cdot \boldsymbol{J}$, we have investigated a set of ECRs. We have identified 116 CLRs (Concentrated Load Regions, $\boldsymbol{E} \cdot \boldsymbol{J}>0$ ) and 35 as CGRs (Concentrated Generator Regions, $\boldsymbol{E} \cdot \boldsymbol{J}<0)$. The automatically selected ECRs have been compared with the overall behaviour of the plasma sheet as described by a reference data base of randomly selected time intervals from the entire Cluster plasma sheet data from 2001.

Our results support the notion that the plasma sheet, on the average, behaves as a load. We observe a larger amount of CLRs than CGRs, and the CLRs appear to be stronger than the CGRs. Analyzing the random reference data we get similar results.

On the other hand, there are investigations which indicate that the picture of the plasma sheet is not entirely that simple. As discussed by Marghitu et al. (2006) and Hamrin et al. (2006), some CGRs in the mid altitude plasma sheet may well be connected to the auroral ionosphere. However, the Cluster apogee is probably too far out at $19 R_{E}$ to catch a possible region at the inner near-Earth plasma sheet where the electromagnetic energy generation for substorm onsets and the aurora generally might take place.
The CLRs are observed closer to the neutral sheet, while the CGRs prefer locations further out, possibly within or close to the PSBL, and the signatures in the magnetic field GSM $B_{x}$ component support this conclusion. This is consistent with results from large scale MHD simulations of energy conversion and transport in the magnetotail (Birn and Hesse, 2005).

Furthermore, from our data we can confirm the importance of the dawn-dusk electric field and cross-tail current for the plasma sheet loads. A majority of the ECRs (both CLRs and CGRs) have $E_{y} J_{y} / \boldsymbol{E} \cdot \boldsymbol{J} \sim 1$, i.e., the GSM $E_{y} J_{y}$ contribution dominates the power density. The GSM $E_{x} J_{x}$ is the smallest one, but GSM $E_{z} J_{z}$ can occasionally be significant. As expected, electric fields and current densities in the positive GSM y-direction (dawn-to-dusk) are most important for CLRs.

The set of automatically selected CLRs and CGRs makes possible a more detailed statistical investigations of the ECRs in the plasma sheet and their general characteristics. For example, in the companion paper H09B we dwell on the issue of the time and length scales of the ECRs. Furthermore, combining the present data with additional information on plasma parameters (like density, bulk flow and temperature), in the future we hope to be able to reveal more of the structure of the plasma sheet.

\section{Appendix A}

\section{Automatic event selection algorithm}

In this article we only consider energy conversion in the plasma sheet. In the plasma sheet the temperature is generally larger than $1 \mathrm{keV}$ and the density smaller than $1 \mathrm{~cm}^{-3}$. Hence, in order separate the plasma sheet events from those in the boundary layer near the magnetopause, we require that the ratio between the proton temperature and density is larger than $1000 \mathrm{eV} / \mathrm{cm}^{-3}$. The choice of this plasma sheet threshold value was verified by visual inspection.

The event selection is based on re-sampling all the data to $4 \mathrm{~s}$. As discussed in Sect. 2, the automatic event selection is based on three separate steps: 1. Selection, 2. Merging, and 3. Rejection. In the first step, ECRs with large enough slopes in the time integral of the power density $\boldsymbol{E} \cdot \boldsymbol{J}$ along the spacecraft path are identified. In the next step neighbouring CLRs are merged as well as neighbouring CGRs. In the final steps, ECRs which do not fulfill a set of physical and instrumental requirements are rejected. Each individual step is explained more throughly below:

\section{Selection:}

(a) The minimum slope required in the integral of the power density is $0.4 \mathrm{pW} / \mathrm{m}^{3}\left(-0.4 \mathrm{pW} / \mathrm{m}^{3}\right)$ for CLRs (CGRs). Hence, weak ECRs are discarded. 
(b) The value of the step size in the integrated power density should at least be $200 \mathrm{pJ} / \mathrm{m}^{3}\left(-200 \mathrm{pJ} / \mathrm{m}^{3}\right)$ for CLRs (CGRs). This is based on the examination of the ECR events in the database used by Marghitu et al. (2009), showing that for a time resolution of $24 \mathrm{~s}$, the step size of the smallest ECRs is about $10 \mathrm{pW} / \mathrm{m}^{3}$. To compare with our threshold this value should be multiplied with 24 , resulting in a minimum step size of $240 \mathrm{pJ} / \mathrm{m}^{3}$, i.e. just above our threshold.

(c) We allow for somewhat noisy ECRs. For each ECR we therefore allow approximately $10 \%$ of the $4 \mathrm{~s}$ steps to fail the step size threshold. However, we require that no ECR starts or ends with such penalty steps.

\section{Merging:}

(a) If the gap in time between two selected and neighboring ECRs of the same type (either two CLRs or two CGRs) is shorter than the shortest ECRs, the two are merged together.

(b) The merging is iterated twice so that several ECRs can be merged into longer events.

3. Rejection:

(a) To ensure reliable estimates from the curlometer method, the elongation and planarity of the Cluster tetrahedron should be smaller than 0.4 (Robert et al., 1998).

(b) To ensure that ions behave collectively, the spatial scale of the ECR obtained as as $\Delta T V_{p l}$ (where $\Delta T$ is the time extent of the ECR and $V_{p l}$ is the average plasma flow within the ECR) should be larger than 5 proton gyroradii.

(c) The ECR should at least consist of $100 \mathrm{~s}$ of data, i.e., more than 25 data points sampled every $4 \mathrm{~s}$. This guarantees statistical reliability of the data.

(d) To ensure that all spacecraft observe the same ECR structure, the time for the plasma to flow through the Cluster tetrahedron, $L / V_{p l}$ (where $L$ is the characteristic size of the tetrahedron and $V_{p l}$ is the average plasma flow within the ECR), should be a few times, $N$, larger than the time extent of the ECR. In this investigation we used $N=5$.

(e) Data from both HIA and CODIF must exist.

(f) The power density obtained by using electric field estimates from CODIF and HIA, respectively, must correlate within the ECR. The correlation coefficient should be more than 0.4 , which corresponds to a moderate correlation or better.

(g) If EFW data exist, the power density obtained by using electric field estimates from CODIF and
EFW, respectively, should correlate. However, the EFW electric field is only available in the satellite spin plane, i.e., in the DSI xy-plane. We only check the correlation between the contributions from the DSI $y$ direction, $E_{y} J_{y}$, obtained by EFW and CODIF, respectively. The correlation coefficient should be more than 0.4.

(h) To ensure good statistics from the CIS instruments and avoid data from the lobes, the average density within the ECR must be at least $0.3 \mathrm{~cm}^{-3}$.

(i) To remove all ECRs that might be afflicted too much with noise, we compare the selected ECRs with 5 equally long (in time) data sets just before the ECRs and 5 equally long data sets just after, i.e., in total 10 reference data sets. The average of $|\boldsymbol{E} \cdot \boldsymbol{J}|$ within the selected ECR should be at least twice as large as in five or more of the reference sets.

The results from the automatic selection routine have been analyzed manually with satisfactory results. However, a couple of ECRs, with rather undetermined power density signatures, have been removed from the statistics.

Acknowledgements. We thank M. André and the EFW team for providing electric field data, the CIS team for the ion data, and the FGM team for the magnetic field data. O.M. acknowledges support through the PECS contract ECSTRA, C98048, and the kind hospitality of Max-Planck-Institut für extraterrestrische Physik, Garching.

Topical Editor R. Nakamura thanks G. Rostoker and J. Vogt for their help in evaluating this paper.

\section{References}

Balogh, A., Dunlop, M. W., Cowley, S. W. H., Southwood, D. J., Thomlinson, J. G., Glassmeier, K. H., Musmann, G., Luhr, H., Buchert, S., Acuna, M. H., Fairfield, D. H., Slavin, J. A., Riedler, W., Schwingenschuh, K., and Kivelson, M. G.: The Cluster magnetic field investigation, Space Sci. Rev., 79, 65-91, 1997.

Birn, J. and Hesse, M.: Details of current disruption and diversion in simulations of magnetotail dynamics, J. Geophys. Res., 101, 15345-15358, 1996.

Birn, J. and Hesse, M.: Energy release and conversion by reconnection in the magnetotail, Ann. Geophys., 23, 3365-3373, 2005, http://www.ann-geophys.net/23/3365/2005/.

Birn, J., Hesse, M., and Schindler, K.: MHD simulations of magnetotail dynamics, J. Geophys. Res., 101, 12939-12954, 1996.

Borovsky, J. E.: Auroral arc thicknesses as predicted by various theories, J. Geophys. Res., 98, 6101-6138, 1993.

Dunlop, M. W., Balogh, A., Glassmeier, K. H., and Robert, P.: Four-point Cluster application of magnetic field analysis tools: The Curlometer, J. Geophys. Res., 107, 1384, doi:10.1029/2001JA005088, 2002.

Escoubet, C. P., Fehringer, M., and Goldstein, M.: Introduction The Cluster mission, Ann. Geophys., 19, 1197-1200, 2001, http://www.ann-geophys.net/19/1197/2001/. 
Gustafsson, G., Bostrom, R., Holback, B., Holmgren, G., Lundgren, A., Stasiewicz, K., Ahlen, L., Mozer, F. S., Pankow, D., Harvey, P., Berg, P., Ulrich, R., Pedersen, A., Schmidt, R., Butler, A., Fransen, A. W. C., Klinge, D., Thomsen, M., Falthammar, C. G., Lindqvist, P. A., Christenson, S., Holtet, J., Lybekk, B., Sten, T. A., Tanskanen, P., Lappalainen, K., and Wygant, J.: The electric field and wave experiment for the Cluster mission, Space Sci. Rev, 79, 137-156, 1997.

Gustafsson, G., André, M., Carozzi, T., Eriksson, A. I., Fälthammar, C.-G., Grard, R., Holmgren, G., Holtet, J. A., Ivchenko, N., Karlsson, T., Khotyaintsev, Y., Klimov, S., Laakso, H., Lindqvist, P.-A., Lybekk, B., Marklund, G., Mozer, F., Mursula, K., Pedersen, A., Popielawska, B., Savin, S., Stasiewicz, K., Tanskanen, P., Vaivads, A., and Wahlund, J.-E.: First results of electric field and density observations by Cluster EFW based on initial months of operation, Ann. Geophys., 19, 1219-1240, 2001, http://www.ann-geophys.net/19/1219/2001/.

Hamrin, M., Marghitu, O., Rnnmark, K., Klecker, B., Andr, M., Buchert, S., Kistler, L. M., McFadden, J., Rme, H., and Vaivads, A.: Observations of concentrated generator regions in the nightside magnetosphere by Cluster/FAST conjunctions, Ann. Geophys., 24, 637-649, 2006, http://www.ann-geophys.net/24/637/2006/.

Haerendel, G.: Poleward arcs of the auroral oval during substorms and the inner edge of the plasma sheet, J. Geophys. Res., 114, A06214, doi:10.1029/2009JA014138, 2009.

Lu, G., Brittnacher, M., Parks, G., and Lummerzheim, D.: On the magnetospheric source regions of substorm-related field-aligned currents and auroral precipitation, J. Geophys. Res., 105, 1848318493, 2000.

Lysak, R. L.: Auroral electrodynamics with current and voltage generators, J. Geophys. Res., 90, 4178-4190, 1985.

Marghitu, O., Hamrin, M., Klecker, B., Rönnmark, K., Buchert, S., Kistler, L. M., André, M., and Remè, H.: Cluster observations of energy conversion regions in the plasma sheet, CAABook 026, in press, 2009.
Marghitu, O., Hamrin, M., Klecker, B., Vaivads, A., McFadden, J., Buchert, S., Kistler, L. M., Dandouras, I., André, M., and Rème, H.: Experimental investigation of auroral generator regions with conjugate Cluster and FAST data, Ann. Geophys., 24, 619-635, 2006, http://www.ann-geophys.net/24/619/2006/.

Paschmann, G., Quinn, J. M., Torbert, R. B., Vaith, H., McIlwain, C. E., Haerendel, G., Bauer, O. H., Bauer, T., Baumjohann, W. Fillius, W., Förster, M., Frey, S., Georgescu, E., Kerr, S. S., Kletzing, C. A., Matsui, H., Puhl-Quinn, P., and Whipple, E. C.: The Electron Drift Instrument on Cluster: overview of first results, Ann. Geophys., 19, 1273-1288, 2001, http://www.ann-geophys.net/19/1273/2001/.

Rème, H., Aoustin, C., Bosqued, J. M., Dandouras, I., Lavraud, B., et al.: First multispacecraft ion measurements in and near the Earth's magnetosphere with the identical Cluster ion spectrometry (CIS) experiment, Ann. Geophys., 19, 1303-1354, 2001, http://www.ann-geophys.net/19/1303/2001/.

Robert, P., Dunlop, M. W., Roux, A., and Chanteur, G.: Accuracy of current density determination, Chap. 16 in: Analysis methods for multi-spacecraft data, edited by: Paschmann, G. and Daly, P. W., ISSI Scientific Report SR-001, ISSI/ESA, Bern, 1998.

Rosenqvist, L., Buchert, S., Opgenoorth, H., Vaivads, A., and Lu, G.: Magnetospheric energy budget during huge geomagnetic activity using Cluster and ground-based data, J. Geophys. Res., 111, A10211, doi:10.1029/2006JA011608, 2006.

Rostoker, G. and Boström, R.: Mechanism for driving gross Birkeland current configuration in auroral oval, J. Geophys. Res., 81, 235-244, 1976.

Vogt, J., Haerendel, G., and Glassmeier, K. H.: A model for the reflection of Alfven waves at the source region of the Birkeland current system: The tau generator, J. Geophys. Res., 104, 269278, 1999. 